\title{
Distribution of lymphatic tissues and autonomic nerves in supporting ligaments around the cervix uteri
}

\author{
JIANPING ZHANG ${ }^{1}$, LANLAN FENG ${ }^{1}$, YI LU ${ }^{1}$, DONGXIA GUO ${ }^{1}$, TENGTENG XI ${ }^{1}$ and XIAOCHUN WANG ${ }^{2}$ \\ ${ }^{1}$ Department of Obstetrics and Gynecology, The First Affiliated Hospital of Soochow University, Suzhou, Jiangsu 215006; \\ ${ }^{2}$ Department of Anatomy, Medical College, Soochow University, Suzhou, Jiangsu 215123, P.R. China
}

Received November 13, 2012; Accepted February 19, 2013

DOI: $10.3892 / \mathrm{mmr} .2013 .1360$

\begin{abstract}
To investigate the distribution of lymphatic tissues and nerves in the supporting ligaments around the cervix uteri for their tomographical relationship, 9 adult female cadavers were used in this study. Following the incision of all supporting ligaments around the cervix, hematoxylin and esosin (H\&E) and immunohistochemical staining of various sections of these ligaments was performed to enable the distribution of lymph tissues and autonomic nerves to be observed. Four lymph nodes were identified in three cadaver specimens. Three lymph nodes were present at a distance of $2.0 \mathrm{~cm}$ from the cervix in the cranial side of the cardinal ligaments (CLs), and one lymph node was located at a distance of $4.0 \mathrm{~cm}$ from the cervix in the cranial side of the uterosacral ligament (USL). The lymphatic vessels were dispersed in the CLs, scattered in the cervical side of the USLs, and occasionally distributed in the vesicouterine ligaments (VULs). In the CLs, parasympathetic nerves were located at the pelvic lateral wall and went downwards and medially into the cervix, while sympathetic fibers were located in the middle and lower parts of the ligaments. In the USLs, the autonomic nerves, which consisted primarily of sympathetic fibers, went downwards and laterally from the pelvic wall to the cervix. In the VULs, parasympathetic and sympathetic nerves were located in the inner sides of the vesical veins in the deep layers of the ligaments. It is concluded that there are few lymphatic tissues in the supporting ligaments around the cervix uteri, and that nerve-sparing radical hysterectomy (NSRH) may be a safe method for the treatment of early-stage cervical cancer.
\end{abstract}

Correspondence to: Dr Xiaochun Wang, Department of Anatomy, Medical College, Soochow University, 199 Ren-ai Road, Suzhou Industrial Park, Suzhou, Jiangsu 215123, P.R.China

E-mail: sudawangxcmd@yahoo.com.cn

Key words: cervix uteri, ligament, immunohistochemistry, nerve-sparing radical hysterectomy

\section{Introduction}

Carcinoma of the cervix is the second most common cancer among women worldwide. During 2009, 11,270 new cases of invasive cervical cancer and $\sim 4,070$ cervical cancer-related mortalities occurred in the US (1).

Radical hysterectomy (RH) has been the treatment of choice for early-stage cervical carcinomas for more than a century, with an overall 5-year survival rate of $>80 \%(2-4)$. However, it is well known that the classical RH may be accompanied by early and late postoperative morbidity, such as bladder dysfunction, anorectal mobility disorders and sexual dissatisfaction, which seriously influence the quality of life (QOL) of surviving patients. These complications are believed to be the results of surgical injury involving the autonomic innervation of the pelvic organs (5-8). The nerve-sparing technique, originally developed in Japan by Okabayashi in 1921 and subsequently modified by other authors $(3,7,8)$, had been considered as the solution to these complications. Since then, the anatomy of the pelvic autonomic nerves has been verified by open surgery, and the rapid recovery of bladder function following nerve-sparing radical hysterectomy (NSRH) has also been demonstrated. Although the autonomic nerves of the pelvic organs and their origins are well-described in anatomy textbooks, these structures are rarely visualized in operating rooms during surgery, and no consensus has been reached on which parts of the uterine supporting ligaments [cardinal ligament (CL), uterosacral ligament (USL) or vesicouterine ligament (VUL)] should be targeted to minimize the potentially damaging effects of NSRH.

Promising results have been reported regarding the disease-free and overall survival (OS) following NSRH for cervical cancer $(9,10)$. However, concerns remain regarding the radicality and safety of this method. It has been suggested that parametrial lymph nodes may remain within the uterine supporting ligaments following NSRH, which may threaten the radicality of the procedure. Some researchers have suggested that the parametrial lymph nodes distributed in these tissues may cause early tumor spread, since fatty tissue in the lateral parts of the paracervix are not completely removed following NSRH $(3,4,11)$. However, it has been reported that parametrial involvement in RH specimens was observed in $<1 \%$ of patients with early-stage cervical cancer, and so extensive parametrectomy was not recommended $(12,13)$. Whether or not lymph 
nodes are located in the supporting ligaments around the cervix is controversial, and a deep knowledge of the anatomical relationship between lymphatic tissues and pelvic nerve components, with particular reference to NSRH techniques, is not available. Tailored surgery has become a major issue in modern cervical cancer surgery. It is our opinion that filling this gap may significantly help surgeons to choose the most appropriate surgical intervention for each patient.

In the present study, the distribution of lymphatic tissues, autonomic nervous fibers and sympathetic fibers in the supporting ligaments around the cervix were investigated in a series of adult female cadavers and the topographical association between them was described.

\section{Materials and methods}

Materials. Nine formalin-fixed donated adult female cadavers with no evidence of previous pelvic operations were used and macroscopic dissection of normal uterine cervix was performed at the Institute of Anatomy of Suzhou University (Suzhou, China) between December 2008 and December 2010. All the cadavers were adults; the mean age at death was 69.1 \pm 7.4 years (range, 52-81 years). The study was approved by the ethics committee of Soochow University.

Dissection procedures. Firstly, the parietal peritoneum was opened. The peritoneum of the vesicouterine pouch was then incised. The bladder was separated from the central wall of the cervix down to the cranial level of the trigone of the urinary bladder. The peritoneum on the pouch of Douglas and posterior peritoneal leaves of the broad ligament were transversely incised. The connective tissue on the vaginal wall was separated from the central wall of the rectum. Then, the pararectal and paravesical spaces were developed. Following separation of the paravesical and pararectal spaces on each side, the CL (between the pararectal and paravesical space), USL (between the pararectal space and rectovaginal ligament) and VUL (between the paravesical space and vesicocervical space) were isolated. These ligaments were then cut starting from their origin at the uterine cervix.

Immunohistochemistry. The mean lengths of the CLs, USLs and VULs were $4.66 \pm 0.34,6.04 \pm 0.28$ and $2.32 \pm 0.30 \mathrm{~cm}$, respectively. After labeling the direction, serial macroscopic slices (each $10 \mathrm{~mm}$ ) were cut from the specimens. Following dehydration in graded ethanol and transfer to xylene, the tissues were embedded in paraffin. Three serial slices of $5 \mu \mathrm{m}$, cut at $1-\mathrm{cm}$ intervals, were used for immunohistochemical analysis. One 5- $\mu \mathrm{m}$ slice cut at a 2-mm interval was used for standard hematoxylin and eosin (H\&E) staining. Sections analyzed using immunohistochemistry were heated in a pressure cooker (LD2X-30KBS, Shanghai Shengan Medical Equipment Factory, Shanghai, China) for $2 \mathrm{~min}$ in 0.01-mol/1 citrate buffer ( $\mathrm{pH}$ 6.0) for antigen retrieval and then cooled at room temperature (RT) for $20 \mathrm{~min}$. Following several washes in phosphate-buffered saline (PBS; Sigma, St. Louis, MO, USA), the sections were quenched with $3 \%$ hydrogen peroxide solution for $10 \mathrm{~min}$ to block endogenous peroxidase activity. Following several washes in PBS, the sections were incubated with the first antibody: S100 protein (a general nerve marker,
1:400; Dako Goldbridge, Beijing, China), TH (an antibody against tyrosine hydroxylase, a specific marker of sympathetic fibers, 1:400; Dako Goldbridge) or D2-40 (a specific marker of lymphatic endothelium cells, 1:400; Dako Goldbridge). The incubation with the first antibody had a duration of $2 \mathrm{~h}$. Then, all the sections were incubated for $0.5 \mathrm{~h}$ in a Novolink Polymer detection system (PV-9000; Novocastra, Goldbridge, Beijing, China). With diaminobenzidine (DBA) as the chromogen, the sections were briefly counterstained with Mayer's hematoxylin, dehydrated via ethanol, transferred to xylene and coverslipped. All the stained sections were assessed by a pathologist.

\section{Results}

$H \& E$ staining. Four lymph nodes were identified in three cadavers. One and three lymph nodes were observed in the USLs and CLs, respectively. The former was located in the cranial side of the USL at a distance of $4.0 \mathrm{~cm}$ from the uterine cervix, and the latter were present in the cranial side of the CLs at a distance of $2.0 \mathrm{~cm}$ from the uterine cervix (Fig. 1A and B). No lymph nodes were identified in the VULs. Lymph nodes in the CLs were present bilaterally only in one cadaver.

\section{Immunohistochemistry}

Lymphatic vessels. The features of the lymphatic vessels in all the ligaments were similar. The lumen was large and irregular and the wall was thin and intermittent. There were large spaces between the endothelial cells and the basement membrane was not complete. The lymphatic vessels were dispersed in the CLs (Fig. 1C), scattered in the cervical side of the USLs, and occasionally distributed in the VULs.

Autonomic nervous and sympathetic fibers. Autonomic nerves consist of sympathetic and parasympathetic fibers. $\mathrm{S} 100$ is a general nerve marker and $\mathrm{TH}$ is a specific marker of sympathetic fibers, which are both expressed in the cytomembrane, cytoplasm and nucleus of Schwann cells.

In CLs, S100-positive fibers were located in the middle part at a distance of $4.5 \mathrm{~cm}$ from the cervix (Fig. 2A), in the middle and inferior parts at $3.0 \mathrm{~cm}$, close to the ventral side at $2.0 \mathrm{~cm}$ (Fig. 2B-D) and in the caudal and ventral sides at $0.5 \mathrm{~cm}$ (Fig. 2E). There were no TH-positive fibers located in the ligaments at distances of $4.5,4.0,3.5,3.0,2.5$ or $2.0 \mathrm{~cm}$ from the cervix (Fig. 3A-C). The TH-positive fibers were in the middle of the ligaments at a distance of $1.5 \mathrm{~cm}$ from the cervix (Fig. 3D) and in the caudal side of the ligaments at 1.0 and $0.5 \mathrm{~cm}$ (Fig. 3E). In CLs, parasympathetic nerves were located at the pelvic wall and went downwards and medially into the cervix, while sympathetic fibers were located in the middle and lower parts of the ligaments.

In USLs, S100-positive fibers were located in the medial and top part at a distance of $6.0 \mathrm{~cm}$ from the cervix (Fig. 4A), in the middle and inferior parts and close to the lateral side at 4.5, 3.0 and $1.5 \mathrm{~cm}$ (Fig. 4B-D) and in the caudal and lateral sides at $0.5 \mathrm{~cm}$ (Fig. 4E). TH-positive fibers were located in the medial and cephalic parts of the ligaments at distances of 6.0 and $5.5 \mathrm{~cm}$ from the cervix (Fig. 5A), in the middle and inferior parts of the ligaments at distances of 5.5, 4.5, 4.0, 3.5, 3.0, 2.5 and $2.0 \mathrm{~cm}$ (Fig. 5B-D) and in the caudal and lateral sides of the ligaments at distances of 1.5, 1.0 and $0.5 \mathrm{~cm}$ (Fig. 5E). In USLs, the autonomic nerves, which consisted primarily of 


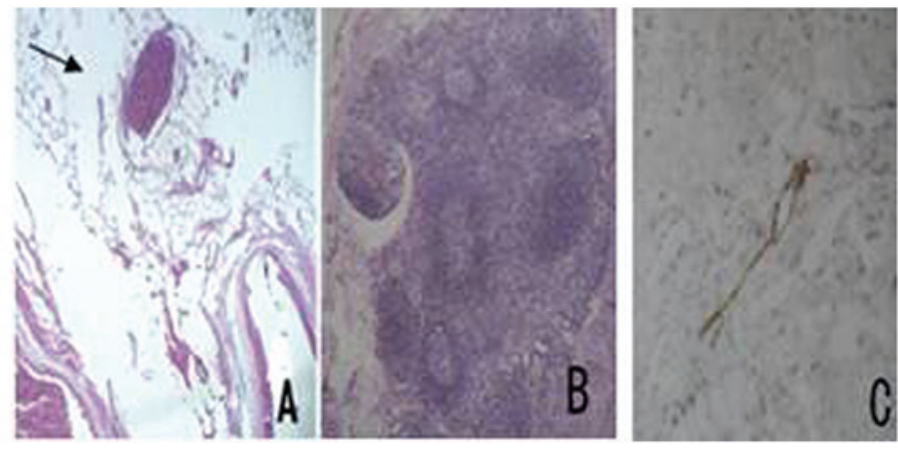

Figure 1. (A) A lymph node located in the cranial side of a cardinal ligament at a distance of $2.0 \mathrm{~cm}$ from the uterine cervix (arrow; magnification, $\mathrm{x} 4$ ) Immunohistochemical staining with TH antibody. The lymph node indicated by the black arrow is enlarged in (B) (magnification, x10). (C) Lymphatic vessel walls were stained brown with anti-D2-40 (magnification, x10).
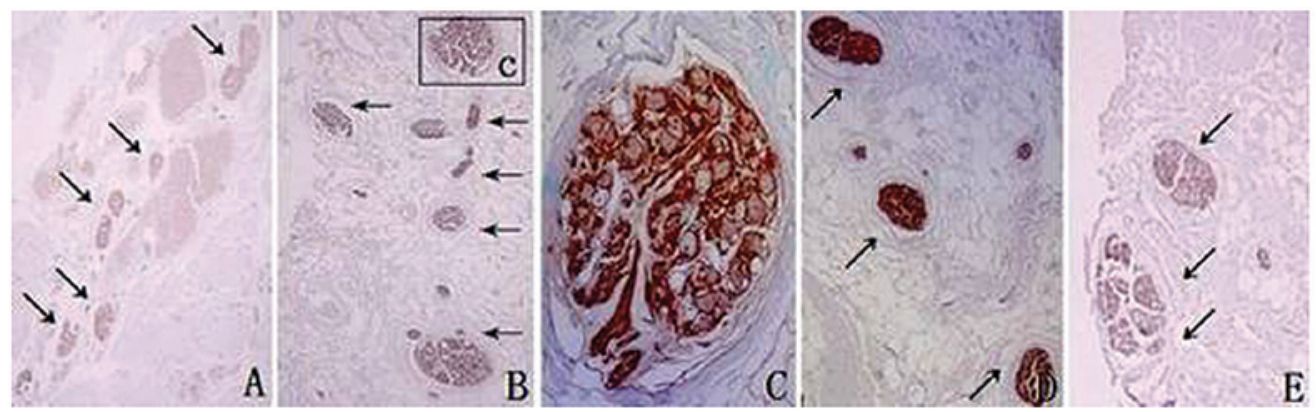

Figure 2. In cardinal ligaments, S100-positive fibers located in the ligaments at distances of (A, right) 4.5, (B, left) 3.0, (D, left) 2.0 and (E, right) $0.5 \mathrm{~cm}$ from the uterine cervix (arrows; magnification, x4). The area indicated by the black rectangle in (B) is enlarged in (C) to show S100-positive fibers (magnification, x10). Immunohistochemical staining with S-100 antibody. Right and left represent right side and left side of human body.
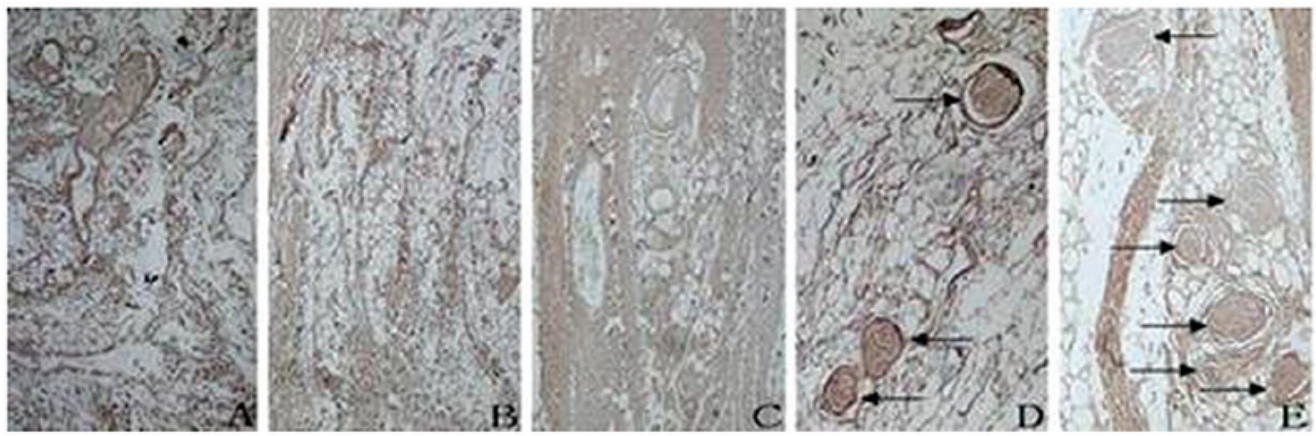

Figure 3. In cardinal ligaments, there were no TH-positive fibers located in the ligaments at distances of (A, right) 4.5 , (B, left) 3.0 and (C, left) $2.0 \mathrm{~cm}$ from the uterine cervix. TH-positive fibers were located in the ligaments at distances of (D, right) 1.5 and (E, left) $1.0 \mathrm{~cm}$ from the uterine cervix (arrows). Magnification, $\mathrm{x} 4$. Immunohistochemical staining with TH antibody. Right and left represent right side and left side of human body.
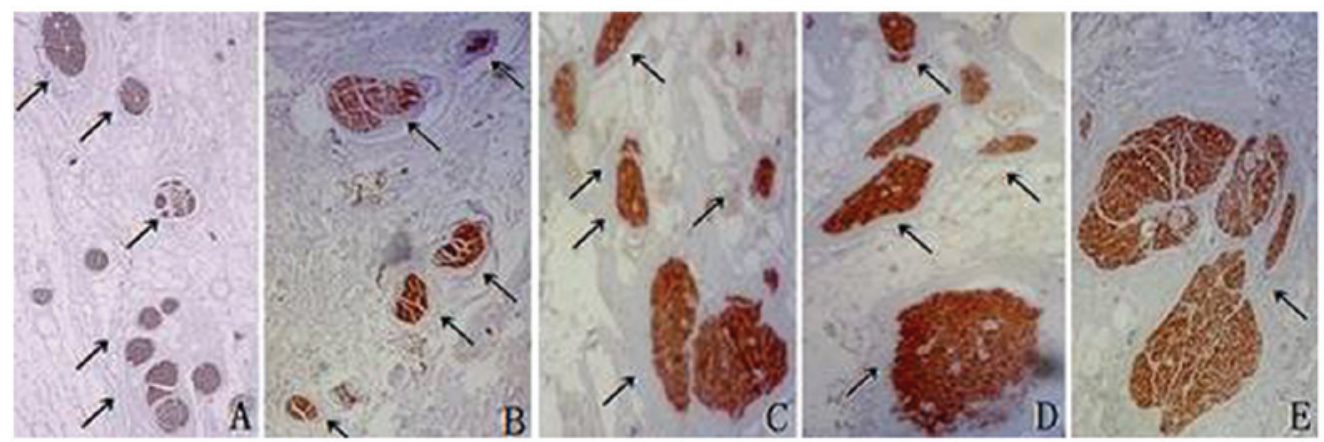

Figure 4. In uterosacral ligaments, S100-positive fibers located in the medial and top parts at distances of (A, left) 6.0, (B, right) 4.5, (C, left) 3.0, (D, right) 1.5 and (E, right) $0.5 \mathrm{~cm}$ from the uterine cervix (arrows). Magnification, x4. Immunohistochemical staining with S100 antibody. Right and left represent right side and left side of human body. 

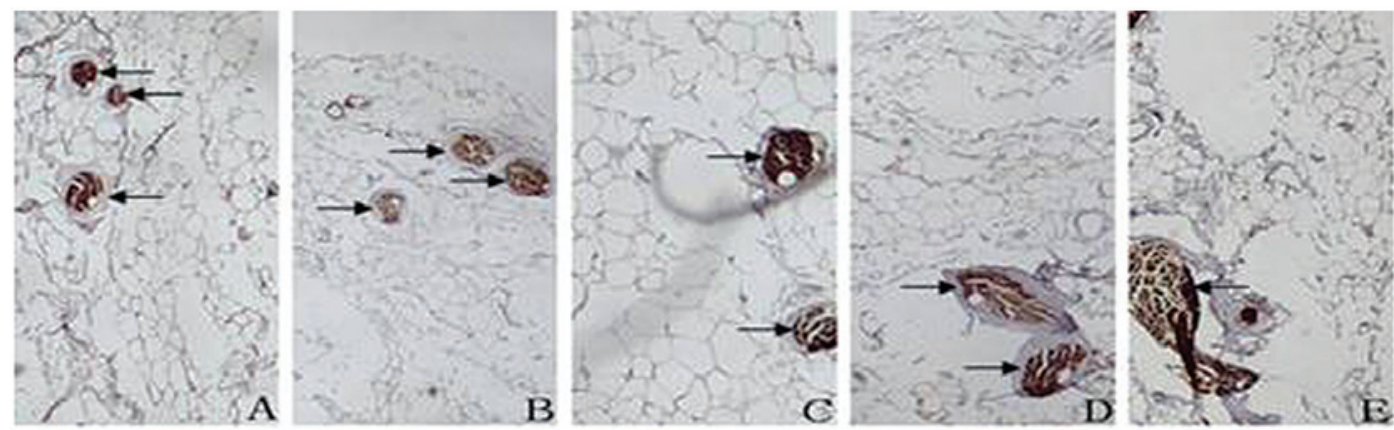

Figure 5. In uterosacral ligaments, TH-positive fibers located in the ligaments at distances of (A, right) 5.5, (B, left) 5.0, (C, left) 3.0, (D, left) 2.0 and (E, right) $0.5 \mathrm{~cm}$ from the uterine cervix (arrows). Magnification, $\mathrm{x} 4$. Immunohistochemical staining with TH antibody. Right and left represent right side and left side of human body.
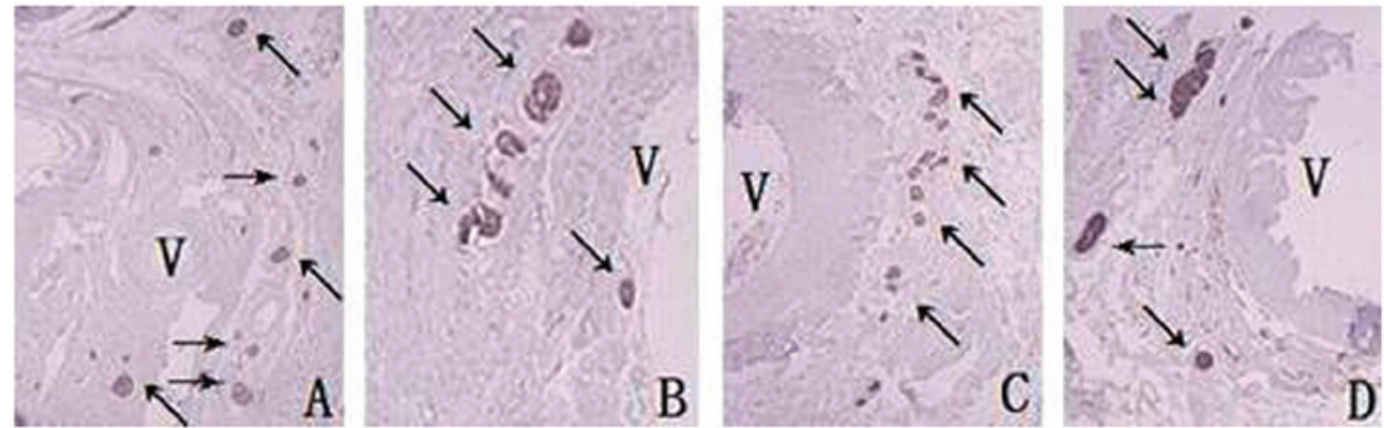

Figure 6. In vesicouterine ligaments, S100-positive fibers located in the medial side of vesical veins (V) in a deep layer of the VULs at distances of (A, left) 2.0, (B, right) 1.5, (C, left) 1.0 and (D, right) $0.5 \mathrm{~cm}$ from the uterine cervix (arrows). Magnification, x4. Immunohistochemical staining with S100 antibody. Right and left represent right side and left side of human body.
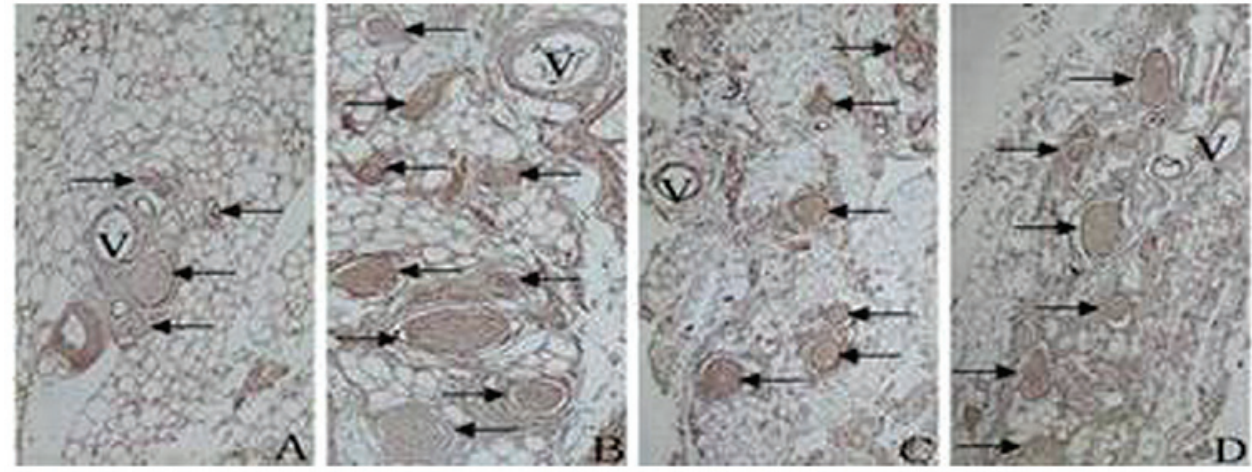

Figure 7. In vesicouterine ligaments, TH-positive fibers located in the medial side of the vesical veins (V) in a deep layer of the VULs at a distance of (A, left) 2.0, (B, right) 1.5 , (C, left) 1.0 and (D, right) $0.5 \mathrm{~cm}$ from the uterine cervix (arrows). Magnification, $\mathrm{x} 4$. Immunohistochemical staining with TH antibody. Right and left represent right side and left side of human body.

sympathetic fibers, went downwards and laterally from the pelvic wall to the cervix.

In the VULs, S100-positive fibers were located in the medial side of the vesical veins in deep layers of the VULs at distances of 2.0, 1.5, 1.0 and $0.5 \mathrm{~cm}$ from the cervix (Fig. 6). TH-positive fibers were located in the medial side of the vesical veins in deep layers of the VULs at distances of 2.0, 1.5, 1.0 and $0.5 \mathrm{~cm}$ from the cervix (Fig. 7). In VULs, the greatest number of parasympathetic and sympathetic nerves were located in the medial side of the vesical veins in deep layers of the ligaments.

\section{Discussion}

Individualization of treatment to reduce therapy-associated early and late morbidity is the current trend in cervical cancer surgery. The main aim of surgery is to minimize injury to the pelvic autonomic innervation. The ideal surgical management of cervical cancer should reduce morbidity without compromising oncological disease control. The novel classification of RH by Querleu and Morrow (Q-M classification) (11) has been proposed to balance curative effects with 
the risk of adverse consequences. It distinguishes between a type $\mathrm{C} 1$ procedure, which corresponds to the nerve-sparing modification, and a type $\mathrm{C} 2$ procedure, which aims for a complete parametrial resection (11). It is well known that the normal function of the pelvic organs depends on autonomic nerves. These autonomic nerves have important functions for orgasm, sexual arousal, urinary functions and anorectal motility. Several studies describing the anatomical background of NSRH based on gross, macroscopic dissections have been published (14-16).

The superior hypogastric plexus contains mostly sympathetic nerves from the abdominal aortic plexus and lumbar ganglia of the sympathetic trunk. The superior hypogastric plexus caudally and laterally leads to the right and left hypogastric nerves. The hypogastric plexus runs through the lateral layer of the USL to the dorsal part of the paracervix in a craniocaudal fashion. Hypogastric nerves bilaterally incorporate the dorsocranial part of the inferior hypogastric plexus. The pelvic plexus is composed of sympathetic fibers from the hypogastric and parasympathetic fibers, arising from the second, third and fourth sacral roots of the spinal cord (pelvic splanchnic nerves). These fibers then run into the inferior pelvic plexus, which is the most important deep neural network of the lateral part of the cervix. The efferent fibers from the pelvic plexus continue to the rectum (rectal fibers), uterus (uterine fibers), rectovaginal ligament ventrally (rectal and vaginal fibers), and finally to the deep VUL. Preservation of the distal part of the inferior hypogastric plexus keeps postoperative morbidity to a minimum, particularly the fine bladder branch located in the deep layer of the VUL lateral and caudal to the ureter.

Butler-Manuel et al (17) quantitatively and qualitatively assessed the nerve content of the uterine-supporting ligaments following radical or simple hysterectomy using immunocytochemistry. They reported that more nerve tissues were present in the USL than in the lateral third of the CL, and more nerve fibers were present in large trunks in the USL than in the CL. According to a recent study by Mantzaris et al (18), fewer nerve fibers were present in the paracervix in specimens from NSRH than in specimens from standard RH. The results of the present study are consistent with those reported previously. Technical details of NSRH using illustrations and step-by-step instructions were provided by Fujii (8). Injuries to the autonomic pelvic nerves may be encountered particularly during the following phases: hypogastric nerves during the resection of the USL; inferior hypogastric plexus during division of the USL and rectovaginal ligaments; pelvic splanchnic nerve during the division of the deep uterine vein in the CL; and the bladder branch from the inferior hypogastric plexus during the resection of the VUL and the paracolpium.

However, whether the modification of NSRH with respect to the uterine supporting ligaments threatens radicality remains to be elucidated. Lymphatic spread is the most important metastatic pathway of cervical cancer. The removal of parametrial tissue has been considered to be of high importance in the treatment of cervical cancer, since tumors may spread to this area through direct microscopic extension or tumor embolization from the primary lesion to lymph nodes embedded in the parametrial tissue (12). The frequency of lymph node metastases in early-stage cervical cancer has been reported to be $0 \%$ in certain studies and as high as $9.7 \%$ in others (19). The high-risk factors of lymph node metastases include large tumor size, cervical stromal invasion to the middle or deep one-third, as well as parametrial involvement and lymphovascular space invasion (LVSI) (20-23). Women with tumors $<2 \mathrm{~cm}$ in the largest diameter, or stromal infiltration less than half of the stroma, or invasion $<10 \mathrm{~mm}$, had a significantly lower risk regarding involvement of the paracervix and pelvic lymph nodes. Isolated parametrial metastases without positive pelvic nodes have been rare $(11,24-27)$. Several studies have shown that $<1 \%$ of patients with early cervical cancer with favorable pathologic characteristics have parametrial involvement, and that the final pathologic specimens of $\sim 60 \%$ of patients who underwent radical trachelectomy contained no residual disease. Wright et al (26) studied 594 patients who underwent $\mathrm{RH}$. Parametrial involvement was observed in 64 patients $(10.8 \%)$ and was associated with advanced grade, deep cervical stromal invasion, LVSI, large tumor size and lymph node metastases. In a subgroup of 270 patients with negative lymph nodes, no LVSI, and tumors $<2 \mathrm{~cm}$, the incidence of parametrial involvement was only $0.4 \%$. Frumovitz et al (12) reported similar findings in 350 women who underwent RH. Parametrial involvement was noted in $7.7 \%$ of patients. Patients with favorable pathological characteristics (such as those previously mentioned) had no parametrial involvement.

Parametrial lymph nodes constitute the first site of extracervical involvement. However, they have been ignored by clinical pathologists due to their small size and special location. During 1996, Benedetti-Panici et al (27) used a giant sections technique, in which the three parametria were spread, the entire specimen including the cervix was processed, and the sections were cut on the coronal plane. Parametrial nodes were found in $65(60 \%)$ of the 109 giant sections. The superficial and deep layers of the VUL, USL and the lateral parametrium revealed the presence of nodes in 13 (33\%), 10 (26\%), $2(5 \%)$ and 28 patients (70\%), respectively. This study showed that parametrial nodes and their metastases were unevenly scattered between the cervix and the pelvic wall, confirming that paracervical tissue constitutes a major route for the lymphatic spread of cervical cancer. These findings confirmed that the lymph from the cervix was drained by three main trunks: the lateral, anterior and posterior. The lateral trunk runs through the lateral parametrium and is the main lymphatic drainage from the uterine cervix. In 2000 , another study by the same group confirmed that there were many lymph nodes in the inner and outer parts of the lateral parametrium as well as lymphatic vessels, both of which may be potential metastatic sites (28).

Sentinel lymph node (SLN) mapping allows perioperative assessment of the condition of the lymph nodes of patients by using patent blue dye and/or radiocolloid injected into the cervix on the day prior to and on the day of surgery. Plentl and Friedman (29) described a typical pattern of lymphatic drainage from the cervix to regional lymph nodes. According to this pattern, the parametrial node is the first draining node in cervical cancer, although it is too close to the cervix to be detected. In previous studies, the most common SLN site was in the topography of the superficial obturator $(30,31)$, while parametrial lymph nodes were rarely found. Ercoli et al (32) used open macroscopic or laparoscopic pelvic dissections in fresh adult female cadavers following lymphatic channel and node staining with Lipiodol dye solution injected into the 
uterine cervix. Stained lymphatic structures were observed into the lateral parametrium, but not in VULs and USLs. The present study confirmed that there are lymphatic channels lateral, anterior and posterior to the normal cervix. The lymphatic vessels were dispersed in the CLs, scattered in the cervical side of USLs, and occasionally distributed in the VULs. There were few lymphatic tissues in the supporting ligaments of the normal cervix.

In the present study, four lymph nodes were identified in three of nine cadaver specimens; the frequency is lower than that reported by Benedetti-Panici et al (27). This discrepancy has several potential explanations. Firstly, our specimens were restricted to the supporting ligaments of the cervix and did not include the extensive parametrium, where lymphatic tissue may be abundantly found. Moreover, a classical morphological study (33) was specifically conducted on the cadavers of newborn infants and children in their first years of life due to the particular abundance of lymphoreticular tissue, which is known to become atrophic with age, while the present study was conducted on the cadavers of old women. It may be hypothesized that the existence of lymph nodes should be considered an exception rather than a rule. Finally, our findings are hardly comparable to those by Benedetti-Panici et al (27), since this earlier study was performed on surgical specimens from cervical cancer patients, while the present study was performed on subjects without evidence of cervical cancer. Further studies are required to gain a better understanding of the anatomy and pathology of the paracervix and pelvic lymph nodes of cervical cancer patients.

In our opinion, there are few lymphatic tissues in the supporting ligaments of the cervix. Comparing the locations of the lymph nodes with those of the nerve components, indicates that the extent of radicality, including CLs, VULs and USLs, is not affected by the preservation of the autonomic nerves. Observational and comparative clinical data have also shown that there is no difference in the local recurrence rate, disease-free and overall survival following NSRH and conventional techniques $(10,11)$. Moreover, with the development of robotic radical techniques, a laparoscopic NSRH may minimize the impairment of radicality by precise neurovascular dissection using the magnified laparoscopic view, direct access with the tip of the laparoscope and the laparoscopic instruments to the deep pelvic structures, and by securing a clear resection margin during the dissection of the deep part of the CLs, USLs, and the posterior leaf of the VULs (34).

In conclusion, NSRH is a safe and feasible method, which does not compromise radicality, and may be considered as the standard treatment for early-stage cervical cancer.

\section{Acknowledgements}

This study was supported by a grant from Jiangsu Province.

\section{References}

1. Jemal A, Siegel R, Ward E,Hao Y, Xu J and Thun MJ: Cancer statistics, 2009. CA Cancer J Clin 59: 225-249, 2009.

2. Piver MS, Rutledge F and Smith JP: Five classes of extended hysterectomy for women with cervical cancer. Obstet Gynecol 44: 265-272, 1974.
3. Raspagliesi F, Ditto A, Hanozet F, Martinelli F, Solima E, Zanaboni F, et al: Nerve-sparing radical hysterectomy in cervical cancer: evolution of concepts. Gynecol Oncol 107: 119-121, 2007.

4. Pluta M, Rob L, Charvat M, Chmel R, Halaska M Jr, Skapa P, et al: Less radical surgery than radical hysterectomy in early stage cervical cancer: a pilot study. Gynecol Oncol 113: 181-184, 2009.

5. Likic IS, Kadija S, Ladjevic NG, Stefanovic A, Jeremic K, Petkovic S, et al: Analysis of urologic complications after radical hysterectomy. Am J Obstet Gynecol 99: 644, 2008.

6. Jackson KS and Naik R: Pelvic floor dysfunction and radical hysterectomy. Int J Gynecol Cancer 16: 354-363, 2006.

7. Yabuki Y, Sasaki H, Hatakeyama N and Murakami G: Discrepancies between classic anatomy and modern gynecologic surgery on pelvic connective tissue structure: harmonization of those concepts by collaborative cadaver dissection. Am J Obstet Gynecol 193: 7-15, 2005.

8. Fujii S: Anatomic identification of nerve-sparing radical hysterectomy: a step-by-step procedure. Gynecol Oncol 111: 33-41, 2008.

9. van den Tillaart SAHM, Kenter GG, Peters AA, Dekker FW Gaarenstroom KN, Fleuren GJ, et al: Nerve-sparing radical hysterectomy: local recurrence rate, feasibility, and safety in cervical cancer patients stage IA to IIA. Int J Gynecol Cancer 19: 39-45, 2009.

10. Espino-Strebel EE, Luna JT and Domingo EJ: Comparison of the feasibility and safety of nerve-sparing radical hysterectomy with the conventional radical hysterectomy. Int J Gynecol Cancer 20: 1274-1283, 2010.

11. Querleu D and Morrow CP: Classification of radical hysterectomy. Lancet Oncol 9: 297-303, 2008.

12. Frumovitz M, Sun CC, Schmeler KM, Deavers MT, Dos Reis R, Levenback CF and Ramirez PT: Parametrial involvement in radical hysterectomy specimens for women with early-stage cervical cancer. Obstet Gynecol 114: 93-99, 2009.

13. Schmeler K, Frumovitz M and Ramirez P: Conservative management of early stage cervical cancer: is there a role for less radical surgery? Gynecol Oncol 120: 321-325, 2011.

14. Mauroy B, Demondion X, Bizet B, Claret A, Mestdagh P and Hurt C: The female inferior hypogastric (=pelvis) plexus: anatomical and radiological description of the plexus and its afferences - applications to pelvic surgery. Surg Radiol Anat 29: 55-66, 2007.

15. Kato T, Suzaka K and Osaki T: Unilateral or bilateral nerve-sparing radical hysterectomy: a surgical technique to preserve the pelvic autonomic nerves while increasing radicality. Int J Gynecol Cancer 17: 1172-1178, 2007.

16. Hazewinkel MH, Sprangers MA, van der Velden J, van der Vaart CH, Stalpers LJ, Burger MP and Roovers JP: Long-term cervical cancer survivors suffer from pelvic floor symptoms: a cross-sectional matched cohort study. Gynecol Oncol 117: 281-286, 2010.

17. Butler-Manuel SA, Buttery LD, A'Hern RP, Polak JM and Barton DP: Pelvic nerve plexus trauma at radical and simple hysterectomy: a quantitative study of nerve types in the uterine supporting ligaments. J Soc Gynecol Investig 9: 47-51, 2002.

18. Mantzaris G, Rodolakis A, Vlachos G, Athanasiou S, Theocharis S, Sotiripoulou ChM and Antsaklis A: Magnifying lenses assisted nerve-sparing radical hysterectomy and prevention of nerve plexus trauma. Int J Gynecol Cancer 18: 868-875, 2008.

19. van Meurs H, Visser O, Buist MR, Ten Kate FJ and van der Velden J: Frequency of pelvic lymph node metastases and parametrial involvement in stage IA2 cervical cancer: a population-based study and literature review. Int J Gynecol Cancer 19: 21-26, 2009.

20. Sakuragi N: Up-to-date management of lymph node metastasis and the role of tailored lymphadenectomy in cervical cancer. Int J Clin Oncol 12: 165-175, 2007.

21. Tavares MB, Sousa RB, Oliveirae Silva T, Moreira LA, Silva LT, Tavares CB and Vieira SC: Prevalence of prognostic factors for cancer of the uterine cervix after radical hysterectomy. Sao Paulo Med J 127: 145-149, 2009.

22. Kasamatsu T, Onda T, Sawada M, Kato T and Ikeda S: Radical hysterectomy for FIGO stage IIB cervical cancer: clinicopathological characteristics and prognostic evaluation. Gynecol Oncol 114: 69-74, 2009

23. Herr D, Konig J, Heilmann V, Koretz K, Kreienberg R and Kurzeder C: Prognostic impact of satellite-lymphovascular space involvement in early-stage cervical cancer. Ann Surg Oncol 16: 128-132, 2009. 
24. Lim CS, Alexander-Sefre F, Allam M, Singh N, Aleong JC, Al-Rawi $\mathrm{H}$ and Jacobs IJ: Clinical value of immunohistochemically detected lymphovascular space invasion in early stage cervical carcinoma. Ann Surg Oncol 15: 2581-2588, 2008.

25. Strnad P, Robova H, Skapa,P, Pluta M, Hrehorcak M, Halaska M and Rob L: A prospective study of sentinel lymph node status and parametrial involvement in patients with small tumour volume cervical cancer. Gynecol Oncol 109: 280-284, 2008

26. Wright JD, Grigsby PW, Brooks R, Powell MA, Gibb RK, Gao F, et al: Utility of parametrectomy for early stage cervical cancer treated with radical hysterectomy. Cancer 110: 1281-1286, 2007.

27. Benedetti-Panici P, Maneschi F, Scambia G, Greggi S, Cutillo G, D'Andrea G, et al: Lymphatic spread of cervical cancer: an anatomical and pathological study based on 225 radical hysterectomies with systematic pelvic and aortic lymphadenectomy. Gynecol Oncol 62: 19-24, 1996.

28. Benedetti-Panici P, Maneschi F, D'Andrea G, Cutillo G, Rabitti C, Congiu M, et al: Early cervical carcinoma: the natural history of lymph node involvement redefined on the basis of thorough parametrectomy and giant section study. Cancer 88: 2267-2274, 2000.

29. Plentl AA and Friedman EA: Lymphatic system of the female genitalia. The morphologic basis of oncologic diagnosis and therapy. Major Probl Obstet Gynecol 2: 1-223, 1971.
30. Vieira SC, Sousa RB, Tavares MB, Silva JB, Abreu BA, Santos LG, et al: Preoperative pelvic lymphoscintigraphy is of limited usefulness for sentinel lymph node detection in cervical cancer. Eur J Obstet Gynecol Reprod Biol 145: 96-99, 2009.

31. Du XL, Sheng XG, Jiang T, Li QS, Yu H, Pan CX, et al: Sentinel lymph node biopsy as guidance for radical trachelectomy in young patients with early stage cervical cancer. BMC Cancer 11: 157, 2011

32. Ercoli A, Delmas V, Iannone V, Fagotti A, Fanfani F, Corrado G, et al: The lymphatic drainage of the uterine cervix in adult fresh cadavers: anatomy and surgical implications. Eur J Surg Oncol 36: 298-303, 2009.

33. Plentl AA and Friedman EA (eds): Clinical significance of cervical lymphatics In: Lymphatic System of the Female Genitalia. The Morphological Basis of Oncologic Diagnosis and Therapy. 1st edition. W.B. Saunders, Philadelphia, pp75-115, 1971.

34. Park NY, Chong GO, Hong DG, Cho YL, Park IS and Lee YS: Oncologic results and surgical morbidity of laparoscopic nerve-sparing radical hysterectomy in the treatment of FIGO stage IB cervical cance. Int J Gynecol Cancer 21: 355-362, 2011. 